Scientific Visualization, 2021, volume 13, number 3, pages 75 - 81, DOI: 10.26583/sv.13.3.08

\title{
Investigation of Artificially Induced Bag-Breakup Fragmentation of Water Surface with Optical Methods
}

\author{
A.A. Kandaurov ${ }^{1}$, D.A. Sergeev², Yu.I. Troitskaya3 ${ }^{3}$ \\ Federal Research Center Institute of Applied Physics of the Russian Academy of Sciences \\ ${ }_{1}$ ORCID: 0000-0002-0014-8887, green.pb@gmail.com \\ 2 ORCID: 000o-0003-4910-3935, daniil@ipfran.ru \\ 3 ORCID: 0000-0002-3818-9211, yuliya@ipfran.ru
}

\begin{abstract}
A paper is devoted to the investigation of the processes of artificially induced bagbreakup type of spray formation phenomenon within wind-wave interaction. The optical methods are considered. Experiments were carried out on the Thermostratified Wind-Wave Tank (TSWiWaT) of the IAP RAS. High-speed video filming with the shadow imaging method demonstrates the possibility of artificial reproduction of all the main stages of this phenomenon, which are also observed for the sporadically occurred ones: inflation of a thin membrane surrounded by a thicker rim, rupture of the membrane leading to the formation of small droplets, fragmentation of the rim with the formation of large droplets. The processing of the images allowed us to estimate typical lifetimes and sizes of membrane for artificial bagbreakup events which turned out to be close to the same parameters for sporadically occurring ones.
\end{abstract}

Keywords: wind, sea, droplets, laboratory modeling, visualization, bag-breakup.

\section{Introduction}

Spray of droplets torn off by the wind from the crests of waves plays an important role in the exchange processes between the atmosphere and the hydrosphere, especially under extreme (stormy) conditions. As was shown in studies [1-3], it strongly influences on momentum, heat and moisture fluxes. A quantitative analysis of the spray characteristics in situ is a complicated problem. That is why for a long time investigations have been carried out in laboratory conditions on the wind-wave facilities when the air flow rate is sufficient for fragmentation of the water surface and formation of spray (see [2-9]). Different experimental methods can be divided on point (see for example [4-6]) and volumetric methods [2, 7-9]. Volumetric methods are based on visualization: illumination with a laser sheet [7] or shadowgraph method [2, 8, 9] with video recording, including high-speed filming, which became very popular. It allows to obtain the data of the spray characteristics and to study in detail the process of its formation both. It should be noted that regardless of the methods used, measuring the characteristics of spray (velocity, size) close to the water surface especially for high and strongly breaking waves caused by severe wind is a very difficult task.

In [9] the combination of multi-view high-speed filming and the shadowgraph visualization within laboratory experiments on the Thermostratified Wind-Wave Tank (TSWiWaT) has helped to study in detail the typical mechanisms leading to the generation of spray and classify them for the first time. The dominant mechanism - the so-called bag-breakup fragmentation was identified based on the comparative analysis of the obtained statistics of the main phenomena leading to the generation of spray. According to laboratory experiments in [9] we can estimate that for the whole range of wind parameters (from calm to severe conditions) the average frequency of sporadic bag-breakup event was only 1 per 1000 frames of record (on average over all wind speeds). This strongly complicates detailed investigation of this 
phenomenon. In this case the artificial initiation of the phenomenon seems proper way to solve the problem. A similar approach was previously used in [10], where a bursting bubbles was artificially generated in a stationary liquid. However, unlike underwater bubbles, the phenomenon of bag-breakup fragmentation can be only simulated under conditions of air flow over the water surface.

This paper describes a specially developed method of artificial initiation of the phenomenon of bag-breakup fragmentation and spray generation of the water surface, intended for use in a laboratory experiment to simulate the wind-wave interaction. The investigations of this phenomenon were carried out using optical methods based on visualization.

\section{Description of experimental setup}

In the experiments carried out in [9], the side view filming for a detailed study of spray generation process and a qualitative analysis of spray characteristics have demonstrated that a three-dimensional wind wave can obscure the region of formation of the bag-breakups. Therefore, firstly we solve the problem of artificially initiating the bag-breakup phenomenon on a smooth water surface without waves. Taking into account that further experiments should be performed on a wavy surface with waves we needed experimental results under similar air flow conditions, i.e. on the same wind-wave facility. The experiments were carried out at the TSWiWaT of IAP RAS. The air flume of total length of $9 \mathrm{~m}$ and cross-section $0.4 \times 0.4 \mathrm{~m}$ with the water depth of $1.5 \mathrm{~m}$. The wind speed on the axis was up to $25 \mathrm{~m} / \mathrm{s}$, which corresponds to the equivalent wind speed of $40 \mathrm{~m} / \mathrm{s}$ recalculating to the standard meteorological height of $10 \mathrm{~m}$ for real conditions. The wind speed in our experiments was measured using a Pitot gauge. In [9] to study the processes of spray generation a side view of the water surface was taken with camera within a special semi-submerged watertight underwater box that was located perpendicular to the flume wall (see fig. $1 \mathrm{a}$ ) at a distance of $7.5 \mathrm{~m}$ from the outlet. This box allows placing the camera below the water level. In this work a scheme of a special (unusual) experiment at TSWiWaT was developed for research on an undisturbed water surface. Facility was completely drained and at the working level of the water surface from the 1st to the 7 th sections a rigid flat plexiglass bottom was installed. A rectangular water reservoir $(20 \times 20 \times 70 \mathrm{~cm}$, aligned along the flume) completely filled with water was inserted into rigid bottom in the middle of the 8th section (see fig. $1 \mathrm{~b}$ ). The surface of the water coincided with the level of the plexiglass bottom.

As it was shown in [9] the bag is blown up by the wind from the surface disturbance - a small elevation (protrusion) of water with typical widths about $1 \mathrm{~cm}$. Thus, we needed to provide the artificial initiation of such a disturbance and ensure high repeatability of its sizes and shape. A special electronic-hydraulic pulse system was developed for this purpose.

Initial disturbance was artificially created by underwater jet from a submerged nozzle. The nozzle was placed in vertical orientation $1.5 \mathrm{~cm}$ under water surface. It is assembled of 5 nickel tubes $20 \times 2 \mathrm{~mm}$, lined up in a row in cross-wind direction. Water entered the nozzle at a pressure of 3 bar via an electronically controlled valve that was opened for a period of $20 \mathrm{~ms}$. Driving signal for the valve was combined with triggers of both cameras in synchronization system, controlled via microcontroller.

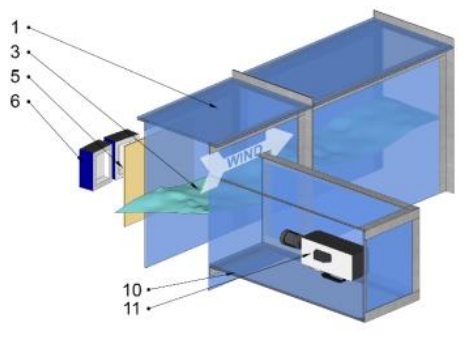

(a)

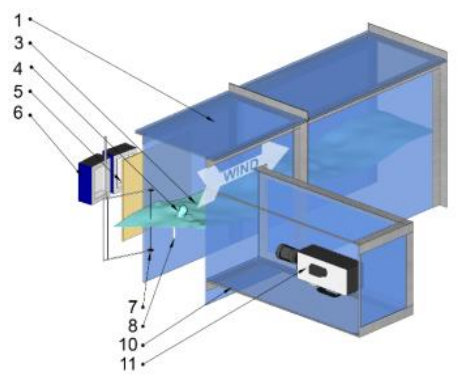

(b)

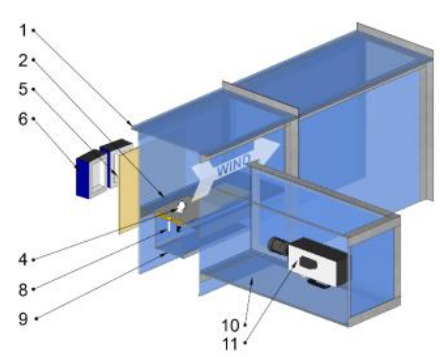

(c)

Fig. 1. Scheme of experiments at TSWiWaT a) usual wind-wave conditions b) with artificial initiation of a disturbance from which a bag is formed for rigid flat surface c) with artificial 
initiation of a disturbance on the wavy surface. Designations: 1) TSWiWaT body 2) rigid surface 3) water surface with waves 4) artificial disturbance on the surface from which the bag-breakup develops 5) opaque screen 6) LED light sources 7) wire wave gauge 8) nozzle of the surface disturbance generating system 9) water reservoir $20 \times 20 \times 70 \mathrm{~cm} \mathrm{10)} \mathrm{watertight} \mathrm{semi-submerged}$ box 11) NAC HX-3 high-speed camera.

\section{System for visualization and image processing}

One of the main requirements for the system of artificial initiation of a disturbance on the surface is the repeatability of its shape. To check this, a high-speed filming of the process of disturbance initiation was performed without air flow in the flume. Similarly to [9], the video was captured from the side by a NAC Memrecam HX-3 high-speed camera installed in a watertight box using a shadow method with illuminating LED lamps located behind the opposite flume wall with an opaque screen at it. The frame rate was 3990 frames per second, exposure 50 $\mu$ s. Each record from the side camera consisted of 700 frames $(175 \mathrm{~ms})$. Resolution was $2560 \times 960$ pixels, dimensions of the filming area $(161 \times 60 \mathrm{~mm})$.

An example of images of the disturbance for different time of evolution is shown in Figure 2a. The visible contour of the disturbance was automatically determined with help of algorithms for image processing taken from the [11], where the form of surface waves with small scale breakings on the crest was determined using Canny method. In Figure $2 \mathrm{~b}$ and $\mathrm{c}$ the time dependences of the width $W$ and heights $H$ of the disturbance on the water surface are shown for ten consecutive cases of initiation performed with a time step of 30 seconds. The $H$ was chosen as the maximal distance from the surface level to the top of the disturbance, and we used a height-averaged width $W$. Time dependencies are plotted from the moment of 60 ms (trigger delay) after valve opening (start of inducing artificial water elevation) until the moment of reaching the maximal $H$ of water surface elevation. Curves are very close to each other, and this can prove that the high repeatability of the form of artificially initiated disturbance created by the developed system.
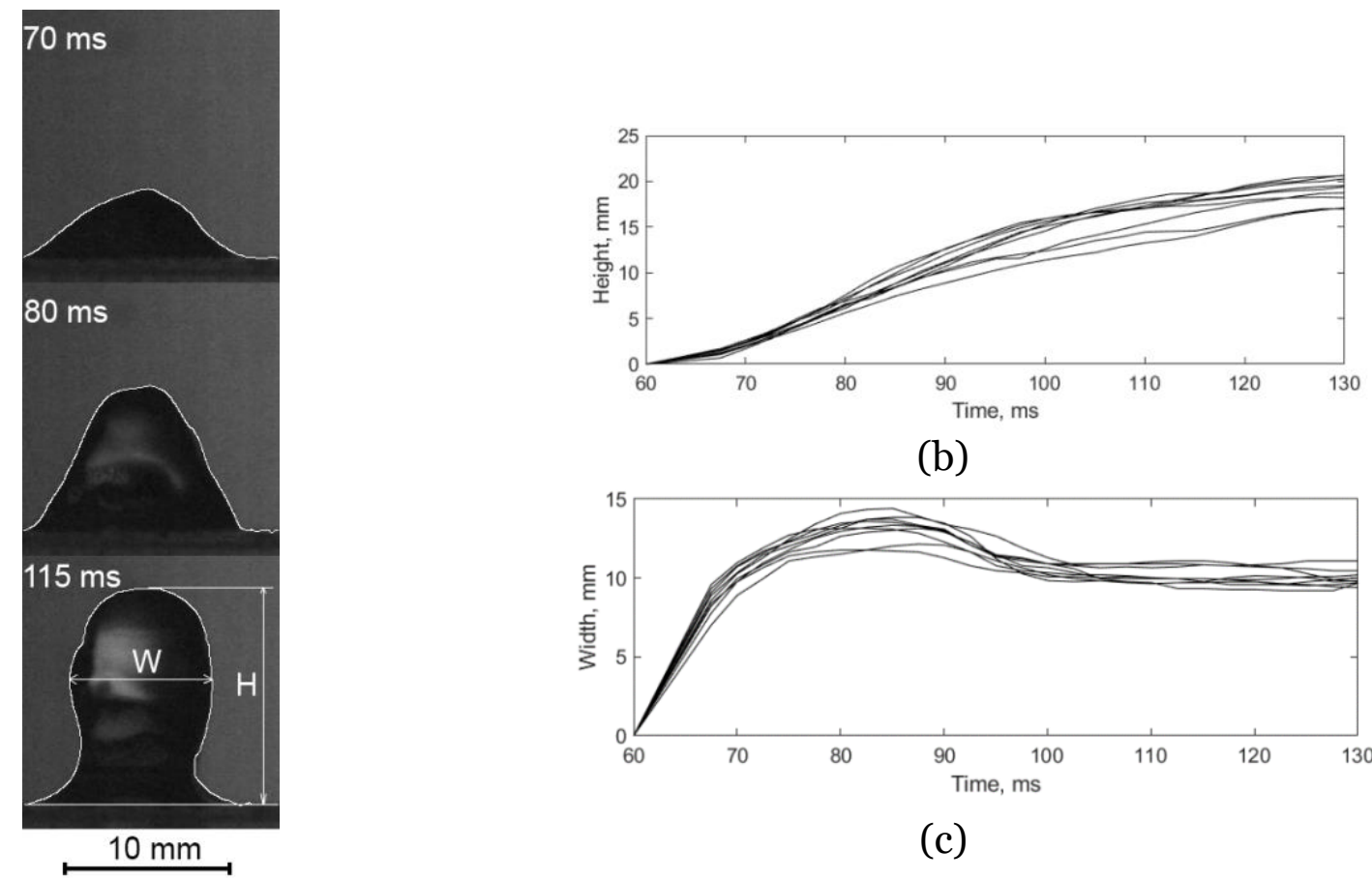

(b)

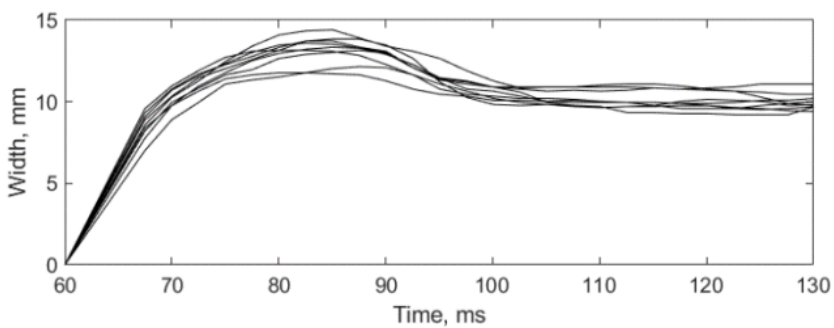

(c)

(a)

Fig. 2. a) Tree consecutive images of an artificially initiated surface disturbance with contour determination and denoting $W$ and $H$ parameters. An automatically determined contour is shown and the height and width are indicated b) Time dependence of the height $H$ for ten successive realizations c) Time dependence of the width $W$ for the same realizations 


\section{Analysis of the obtained data and comparing with previously obtained results.}

The results have demonstrated that the bag-breakups began to form regularly at speeds about $15 \mathrm{~m} / \mathrm{s}$. Fig. 3 shows a comparison of the images obtained in this work, and images of studies of the bag-breakup occurred sporadically in the work [9]. The comparison confirmed that it is possible to reproduce the phenomenon of bag-breakup spray generation using artificial perturbation, including the stages of inflation, membrane rupture, and rim fragmentation. This allowed the further development of the system for conditions of rough surface. Earlier in [9] it was noted that in sporadic regime bag-breakups are mainly formed on the crests of waves. Thus, we had to ensure the operation of the system of artificial initiation of disturbances on the surface at the required phase of the wave (at crests). A resistive wire wave gauge, which located close to the observation area, at a distance of $5 \mathrm{~cm}$ was used to obtain wave parameters (see Fig. 1c). The operation of electronic-hydraulic system was synchronized with adjusted time delay on the basis of signal from wave gauge.
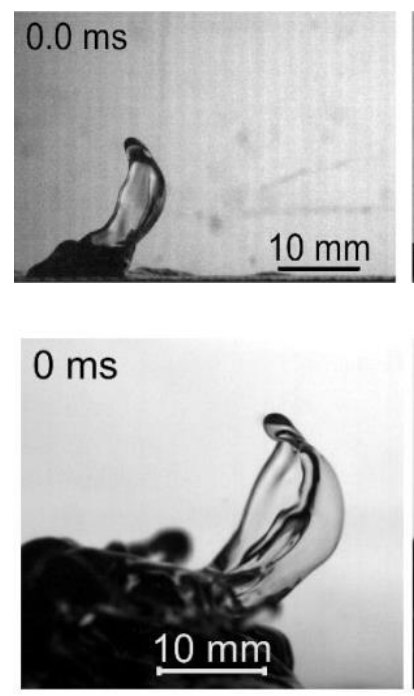

$4 \mathrm{~ms}$

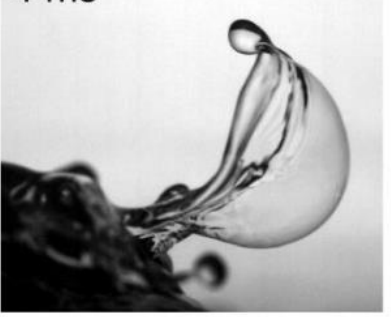

(b)

(a)
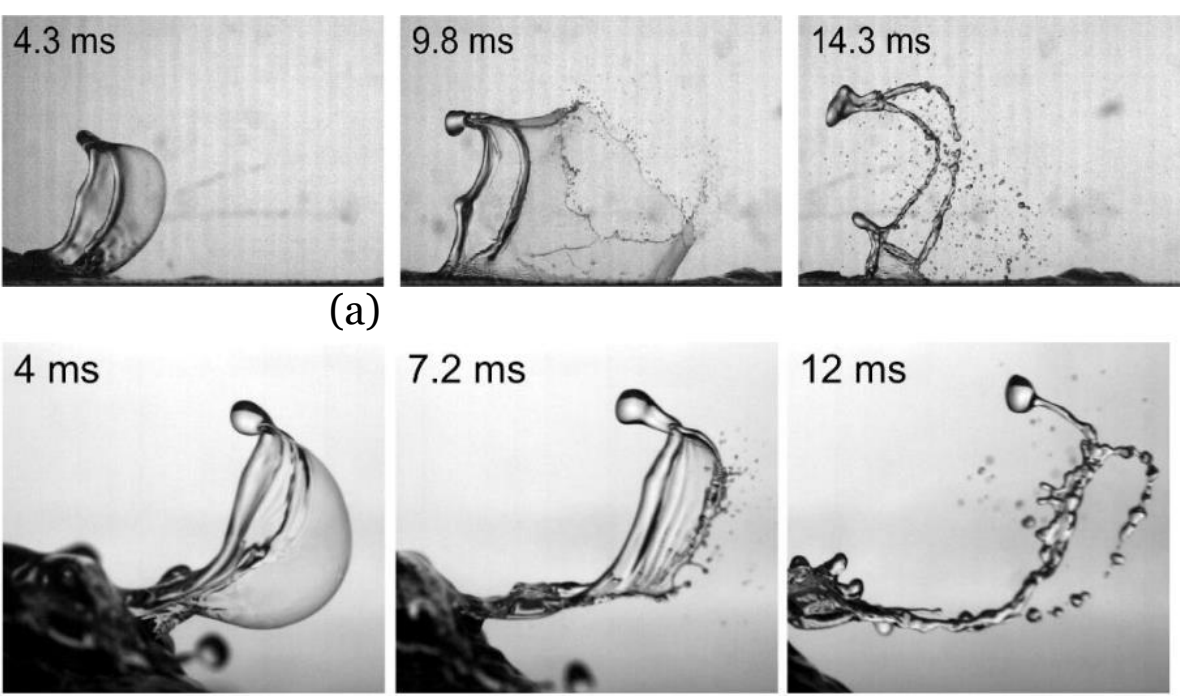

Fig .3. Set of frames of high-speed filming of the formation of a bag-breakup in experiments at TSWiWaT. a) with artificial disturbance of the surface, in the absence of waves according to the results of these studies b) sporadical formation of a bag-breakup taken from [9]

Thus, solely matching of the images was not sufficient and we should quantitatively compare the main characteristics of artificial (in our case) and sporadically occurred bagbreakups. To this purpose we processed the images of the bag-breakups in the manner similar to what proposed in [12] to obtain parameters of maximal size of the membrane before the rupture and lifetime of the bag-breakups. The main difference was that in previous investigation top view images were processed, but in our case the side images. In Figure 4 we see a good agreement between the results. Our data are well fitted with the dependencies of the average values of maximal size and lifetime of the bags on friction velocity $u^{*}$ proposed in [12] within the error bars. 


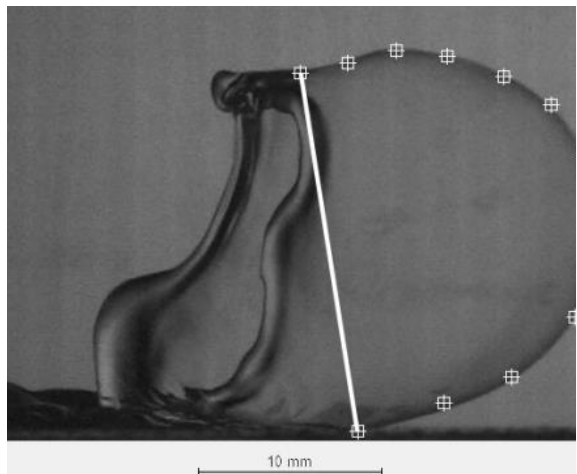

(a)

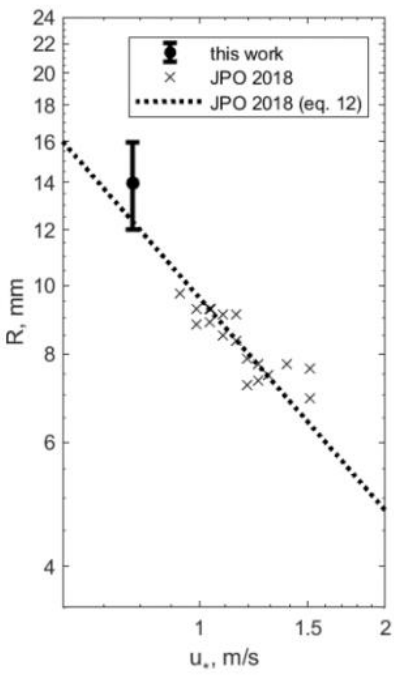

(b)

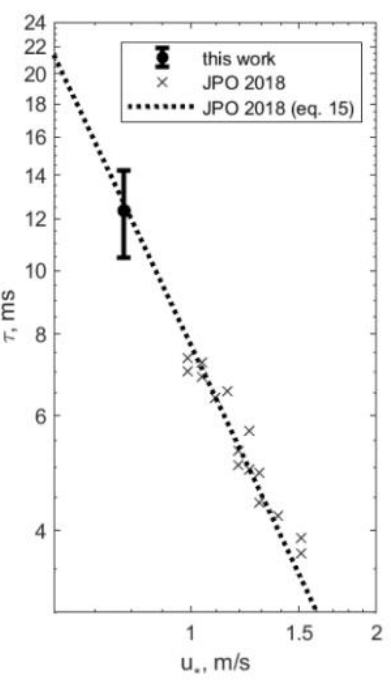

(c)

Fig. 4. a) the image of the bag with illustrating the processing to define the size (radius) of

the bag membrane in the moment of rupture b) comparing our results of determining the averaged radius of bag membrane for the moment of its rupture with data previously obtained in [12] (c) the same comparison for the averaged bag lifetime

\section{Conclusion}

The results of present investigation demonstrated that previously observed sporadic events of bag-breakup fragmentation on the free water surface under the action of air flow could be induced artificially in control time and space area for the condition of laboratory experiments. The shadow imaging method combined with high-speed filming allowed to demonstrate quality and quantity similarity between the artificial and observed in vivo bag-breakup events. It was possible to reproduce all the main stages of this phenomenon: 1) inflation of a thin membrane surrounded by a thicker rim, 2) rupture of the membrane, leading to the formation of small droplets, 3) fragmentation of the rim with the formation of large droplets. Also, special processing of the frame sequences allowed to obtain main integral parameters of bag-breakups: averaged lifetime and maximal size of the membrane before the rupture. It's turned out to be very close to the similar results with obtained for sporadic bag-breakups events. The developed method can be used further for detailed studies of the processes of spray generation due to the phenomenon of bag-breakup fragmentation of the water surface not only at TSWiWaT, but also at other experimental facilities.

\section{Acknowledgement}

This work was supported by the Russian Science Foundation project 19-17-00209 (development of software for image processing) and project 21-19-00755 (development of the hardware part of the artificial disturbance generation system), Russian Foundation Basic Research project 19-05-00249 (development of an optical system), work of A.A. Kandaurov was partially supported by the President's grant for young scientists MK-5503.2021.1.5. The experiments were carried out at unique scientific facility "Complex of large-scale geophysical facilities of IAP RAS".

\section{References}

1. Andreas, E. L., A review of the sea spray generation function for the open ocean// Adv. Fluid Mech., vol. 33, pp. 1-46, 2002.

2. Veron, F., Ocean Spray //Annu. Rev. Fluid Mech., vol. 47, pp. 507-538, 2015. doi.org/10.1146/annurev-fluid-010814-014651 
3. Komori, S. et al., Laboratory Measurements of Heat Transfer and Drag Coefficients at Extremely High Wind Speeds // J. Phys. Oceanogr., vol. 48, pp. 959-974, 2018. doi.org/10.1175/JPO-D-17-0243.1

4. Lai, R. J. \& Shemdin, O. H., Laboratory study of the generation of spray over water // J. Geophys. Res., vol. 79, pp. 3055-3063, 1974. doi.org/10.1029/JCo79io21p03055

5. Wu, J., Spray in the atmospheric surface layer: Laboratory study // J. Geophys. Res., vol. 78, pp. 511-519, 1973. doi.org/10.1029/JCo78ioo3poo511

6. Fairall, C. W., Banner, M. L., Peirson, W. L., Asher, W. \& Morison, R. P., Investigation of the physical scaling of sea spray spume droplet production// J. Geophys. Res., vol. 114, pp. C10001, 2009. doi.org/10.1029/2008JCo04918

7. Anguelova, M., Barber, R. P. and Wu, Laboratory observation of spume drops produced by wind tearing of wave crests // J. Phys. Oceanogr., vol. 29, pp. 1156-1165, 1999. doi:10.1175/1520-0485(1999)029<1156:SDPBTW>2.0.CO;2

8. Koga, M., Direct production of droplets from breaking wind-waves -its observation by a multi-colored overlapping exposure photographing technique // Tellus, vol. 33, pp. 552-563, 1981. doi.org/10.3402/tellusa.v33i6.10776

9. Y. I. Troitskaya, O. S. Ermakova, A. A. Kandaurov, D. S. Kozlov, D. A. Sergeev, and S. S. Zilitinkevich, Fragmentation of the 'bag-breakup' type as a mechanism of the generation of sea spray at strong and hurricane winds // Dokl. Earth Sci., vol. 477, no. 1, pp. 1330-1335, 2017. doi.org/10.1134/S1028334X17110174

10. Lhuissier, H. \& Villermaux, E., Bursting bubble aerosols, J. Fluid Mech., vol. 696, pp. 544, 2012. doi.org/10.1017/jfm.2011.418

11. Kandaurov, A.A., Troitskaya, Y.I., Sergeev, D.A., Vdovin, M.I., Baidakov, G.A. Average velocity field of the air flow over the water surface in a laboratory modeling of storm and hurricane conditions in the ocean // Izvestiya - Atmospheric and Ocean Physics, 2014, vol.50, I.4, pp. 399-410. doi.org/10.1134/Sooo143381404015X

12. Troitskaya, Y. I. Troitskaya, Y.I., Ermakova, O.S., Kandaurov, A.A., Sergeev D.A., Kozlov D.S., Zilitinkevich S.S. Non-monotonous dependence of the ocean surface drag coefficient on the hurricane wind speed due to the fragmentation of the ocean-atmosphere interface// Doklady Earth Sciences, 2017, vol. 477, pp.1373-1378 doi.org/10.1134/S1028334X17110265 